\title{
Chromosomal Aberrations in Chronic Myeloid Leukemia: Response to Conventional TKIs and Risk of Blastic Transformation
}

\author{
Shafaq Maqsood ${ }^{1}$, Fatima Ali², Abdul Hameed ${ }^{3}$, Neelam Siddiqui ${ }^{2}$ \\ ${ }^{1 *}$ Sir Gangaram Hospital, Lahore, Pakistan. ${ }^{2 *}$ Shaukat Khanum Memorial Cancer Hospital and Research Centre, Lahore, Pakistan. \\ $3 *$ Ittefaq Hospital Lahore, Pakistan.
}

\begin{abstract}
Background and Purpose: Chronic Myeloid Leukemia (CML) is a common hematological malignancy. The characteristic molecular abnormality is the presence of Philadelphia chromosome or BCR-ABL fusion gene which is the result of 9:22 translocation. Tyrosine kinase inhibitors (TKIs) form the main stay of treatment in $\mathrm{CML}$ with excellent responses. The purpose of this study was to determine the impact of additional chromosomal abnormalities on outcomes in CML. Methods: This is a retrospective chart review of all patients who were diagnosed with CML in chronic phase (CP) with additional chromosomal abnormalities (ACAs) over a period of 5 years from 2010 to 2015 at Shaukat Khanum Memorial Cancer Hospital and Research Centre, Lahore, Pakistan. Results: A total of 283 patients were diagnosed with CML from January 2010 to January 2015.31 patients out of these were found to have additional chromosomal abnormalities at the time of diagnosis in addition to BCR-ABL fusion gene or Philadelphia chromosome detection. Out of these 31 patients, $23(74.2 \%)$ were males whereas $8(25.8 \%)$ were females. $13(41.9 \%)$ were in the age group of 31 to 50 years whereas the other two groups that is 18 to 30 years and 51 to 70 years had 9 patients each. After approval from the government which usually takes a standard 2-3 weeks' time, these patients were started on tyrosine kinase inhibitors which was Imatinib in $30(96.8 \%)$ and Nilotinib in $1(3.2 \%)$ patient. Conventional cytogenetic analysis performed for each patient at the time of diagnosis revealed that 11 (35.5\%) of patients had variant Philadelphia chromosome followed by 7 patients $(22.6 \%)$ with trisomy 8.5 patients $(16.1 \%)$ had multiple chromosomal abnormalities including trisomy 8, deletion 1 and isochrome 17q. 2 patients each had isochrome 17q, inversion 3 and deletion 9 abnormalities. 1 patient had deletion 7 whereas 1 had variant Philadelphia chromosome with other chromosomal abnormalities. Conclusion: It was evident that frequently occurring ACAs In our CML population were Variant Philadelphia chromosome and trisomy 8 .
\end{abstract}

Keywords: Chronic Myeloid Leukemia- Chronic phase- additional chromosomal abnormalities- blastic transformation

Asian Pac J Cancer Care, 6 (1), 35-39

Submission Date: 08/29/2020Ａcceptance Date: 02/07/2021

\section{Introduction}

Chronic Myeloid Leukemia is a stem cell clonal disease with an annual incidence of 1-1.5 per 100,000 persons. Median age of presentation is around 50 to 60 years in the western part of the world [1]. It is characterized by the translocation between chromosome 9 and 22 resulting in fusion gene BCR-ABL that forms the basis of pathogenecity of CML [2-3]. Philadelphia chromosome was first discovered by Nowell and Hungerford in 1960 and is the cytogenetic hallmark of CML [2- 4]. BCR-ABL fusion gene leads to proliferation of myeloid elements by encoding a protein with tyrosine kinase activity [5]. Most of the patients exhibit BCR-ABL protein which forms the target to tyrosine kinase inhibitors that are the main stay of treatment in CML [4]. The first TKI that was developed and instituted for treating CML was Imatinib mesylate [1].

Corresponding Author:

Dr. Shafaq Maqsood

Senior Registrar Medical Oncology Department, Sir Gangaram Hospital, Lahore, Pakistan.

Email: drshafaq@live.com 
CML can present with or progress through three phases. Chronic phase (CP), accelerated phase (AP) and blast crisis (BP). Most of the patients present in CP [4-5]. Generally, chronic phase is characterized by leukocytosis, anemia and splenomegaly with detection of BCR-ABL fusion gene [6]. Blastic crisis is essentially like acute leukemia and is chemo refractory with a median survival of 3 to 6 months [7]. Secondary chromosomal aberrations are more commonly associated with accelerated and blastic phases of CML [6].

CML is the first malignancy where targeted therapy was introduced based on molecular level. Landmark trial IRIS showed that the first generation TKI Imatinib mesylate led to a complete cytogenetic response (CCyR) in $87 \%$ of the patients. Recent results reported that $17 \%$ of the patients had primary resistance or loss of response (secondary resistance). Treatment failure had been shown to have direct correlation with additional cytogenetic abnormalities (ACAs) which can be interpreted as clonal evolution and chromosomal instability [2-3]. Major cytogenetic aberrations commonly encountered are variant Philadelphia translocation in 5 to 10 percent of patient. Other abnormalities may include an extra Philadelphia chromosome, trisomy 8 , trisomy 19 and isochrome $17 \mathrm{q}$ with loss of p53 or 20q [8]. Therefore, cytogenetic analysis is not only essential to establish the diagnosis of CML and to assess treatment response but also has prognostic significance if additional cytogenetic abnormalities are detected [5].

Clonal evolution occurs in approximately $30 \%$ of CML patients in AP and upto $80 \%$ in blastic phase. Most of the patients in blastic phase have a myeloid phenotype while $25 \%$ show a pre B lymphoblastic phenotype [1]. It has been reported that around $10 \%$ to $12 \%$ of patients with CML in chronic phase have ACAs at the time of diagnosis [9].

First Line TKIs are generally ineffective. Second line TKIs namely Dasatinib and Nilotinib are effective in induction or restoration of complete cytogenetic response in $40 \%$ to $50 \%$ of patients who have not responded to Imatinib in the first place [4].

However, outcome after treatment with second generation TKIs like Dasatinib and Nilotinib has not been extensively studied and more research in this direction is warranted [10].

Our study aims to look at the impact of additional chromosomal abnormalities on survival in patients with CML who were in chronic phase at the time of diagnosis. It also demonstrated the response to conventional TKIs and risk of blastic transformation in the same set of patients while being on therapy.

\section{Materials and Methods}

This was retrospective study in which all patients with the diagnosis of Philadelphia chromosome positive CML$\mathrm{CP}$ with additional chromosomal aberrations present at the time of diagnosis were enrolled. Data collection was from registered patients over a period of 5 years from January 2010 to January 2015. A standard proforma was used for data collection (included patient characteristics, BCL-ABL status, baseline complete blood picture, baseline cytogenetics, disease response, transformation to acute leukemias and outcomes). Disease response was evaluated by hematological response, molecular response and complete cytogenetic response and time to achieve each response.

This study was approved by the Institutional Ethical Committee. The diagnosis of CML was established on the basis of bone marrow biopsy. Cytogenetic analysis and PCR for BCR-ABL were performed in all patients. All Patients 18 years and older with Philadelphia chromosome positive CML-CP with ACAs at the time of diagnosis were included. Patients with CML whose cytogenetic and molecular analysis could not be done at the time of initial presentation and Patients in Accelerated phase and blastic phase at the time of diagnosis were excluded.

Following definitions were used throughout the study: Complete hematological response was defined as normalization of peripheral blood counts with total leukocyte less than $10 \times 109 / \mathrm{L}$, platelets less than $450 \times$ 109/L, no immature cells and disappearance of palpable splenomegaly, complete cytogenetic response was defined as no Philadelphia positive metaphases, and Molecular response was defined as no detected BCR-ABL using a PCR assay.

Descriptive analysis was done for both categorical and continuous variables. These included ages, gender, presenting phase of CML, BCR-ABL status at diagnosis (detected or not detected), first line TKI chosen with doses, time to achieve complete hematological, cytogenetic and molecular response, switch to alternate therapy, transformation to acute leukemia, therapy instituted in case of transformation and outcomes both with and without transformation. The outcome of interest was cHR, CcyR and CMR with first generation TKIs in patients with aberrant chromosomal abnormalities. Secondary outcome was the risk of transformation associated with ACA. Tertiary outcome was the patient survival with transformation.

Age groups were subdivided into 18 to 30 years, 31 years to 50 year and 51 to 70 years. Time to achieve cHR and $\mathrm{CMR}$ was categorized as within 3 months, 3 to 6 months, 6 to 12 months, more than 12 months and never achieved where time to achieve CcyR was described as within 6 months, 6 to 12 months, 12 to 24 months, more than 24 months and never achieved.

\section{Results}

A total of 283 patients were diagnosed with CML from January 2010 to January 2015 and their data was accessed through Hospital Information System. 31 patients out of these were found to have additional chromosomal abnormalities at the time of diagnosis, along with BCRABL fusion gene or Philadelphia chromosome detection. Chromosomal abnormalities were detected through conventional cytogenetic analysis. All those patients who had chromosomal abnormalities but were already in blastic phase at presentation were not included in the study. 
Table 1. Characteristics of Patients Diagnosed with CML-CP and with Additional Chromosomal Abnormalities at the Time of Diagnosis, Choice of Initial Tyrosine Kinase Inhibitor and Response Assessment

\begin{tabular}{|c|c|c|}
\hline Patient Characteristics & Number & $\begin{array}{c}\text { Percentage } \\
(\%)\end{array}$ \\
\hline Total Patients with chromosomal aberrations at the time of diagnosis of CML in Chronic phase & 31 & \\
\hline Males $(\mathrm{N}, \%)$ & 23 & 74.20 \\
\hline Females & 8 & 25.80 \\
\hline Age Range(years) & $18-70$ & \\
\hline Minimum Median Follow-up (Months) & 31 months & \\
\hline BCR-ABL detected by Quantitative PCR assay & 31 & 100 \\
\hline \multicolumn{3}{|l|}{ Initial Tyrosine Kinase inhibitor } \\
\hline Glivec & 30 & 96.80 \\
\hline Nilotinib & 1 & 3.20 \\
\hline \multicolumn{3}{|l|}{ Response to initial TKIs Complete Hematological Response } \\
\hline Within 3 months & 16 & 51.60 \\
\hline 3-6 months & 9 & 29 \\
\hline 6-12 months & 4 & 12.90 \\
\hline Never achieved & 2 & 6.5 \\
\hline \multicolumn{3}{|l|}{ Complete Molecular Response } \\
\hline 3-6 months & 5 & 16.10 \\
\hline 6-12 months & 6 & 19.40 \\
\hline$>12$ months & 3 & 9.70 \\
\hline Never achieved & 17 & 54.80 \\
\hline \multicolumn{3}{|l|}{ Complete Cytogenetic Response } \\
\hline Within 6 months & 2 & 6.50 \\
\hline 6-12 months & 4 & 12.90 \\
\hline 12-24 months & 3 & 9.70 \\
\hline$>24$ months & 4 & 12.90 \\
\hline Never achieved & 18 & $58.10 \%$ \\
\hline
\end{tabular}

Out of these 31 patients, $23(74.2 \%)$ were males whereas $8(25.8 \%)$ were females. $13(41.9 \%)$ were in the age group of 31 to 50 years whereas the other two groups that is 18 to 30 years and 51 to 70 years had 9 patients each. After approval from the government which usually takes a standard 2-3 weeks' time, these patients were started on tyrosine kinase inhibitors which was Imatinib in $30(96.8 \%)$ and Nilotinib in $1(3.2 \%)$ patients. Response assessment was by routine complete blood count, BCR-ABL by PCR and 3 monthly interval and first chromosomal analysis at 6 months. It is interesting to note, that $16(51.6 \%)$ patients achieved complete hematological response within 3 months. However a complete molecular and cytogenetic response was never achieved in 17 $(54.8 \%)$ and $18(58.1 \%)$ patients respectively (Table 1$)$.

\section{Cytogenetic Analysis}

Conventional cytogenetic analysis performed for each patient at the time of diagnosis revealed that $11(35.5 \%)$ of patients had variant Philadelphia chromosome followed by 7 patients $(22.6 \%)$ with trisomy 8.5 patients $(16.1 \%)$ had multiple chromosomal abnormalities including trisomy 8 , deletion 1 and isochrome 17q. 2 patients each had isochrome 17q, inversion 3 and deletion 9 abnormalities. 1 patient had deletion 7 whereas 1 had variant Philadelphia chromosome with other chromosomal abnormalities (Table 2).

\section{Risk of transformation to acute leukemia}

Out of 31 patients, there was no transformation to blastic phase in $20(64.5 \%)$ patient. $8(25.8 \%)$ patients transformed to acute myeloid leukemia whereas $3(9.7 \%)$ patients had a transformation to acute lymphoblastic leukemia (Table 3)

\section{Toxicity profile of Imatinib}

No significant toxicity was observed in 9 (29\%) of patients. Major toxicity was cytopenias which was observed in $13(41.9 \%)$ of patients whereas 4 patients had gastrointenstinal side effects. Remaining 4 patients has other toxicity profile like skin rash, edema etc. 1 patient who was put on Nilotinib right from the start did not report any major adverse effects.

\section{Therapy and response in transformed patients}

Out of the 11 patients who transformed to AML and ALL, 6 patients underwent induction chemotherapy while 5 were not considered fit for it on account of poor 
Table 2. Additional Chromosomal Abnormalities

\begin{tabular}{lcc}
\hline Additional chromosomal abnormalities detected by cytogenetic analysis & Number & Percent (\%) \\
\hline Variant Philadelphia chromosome & 11 & 35.5 \\
Trisomy 8 & 7 & 22.6 \\
Isochrome 17q & 2 & 6.5 \\
Inversion 3 & 2 & 6.5 \\
Deletion 9 & 2 & 6.5 \\
Deletion 7 & 1 & 3.2 \\
Variant Philadelphia with additional chromosomal abnormalities & 1 & 3.2 \\
Multiple chromosomal abnormalities (Notably trisomy 8/ isochrome 17q and/or del 1) & 5 & 16.1 \\
\hline
\end{tabular}

Table 3. Risk of Blastic Transformation

\begin{tabular}{lcc}
\hline Patients transformed to acute leukemia & Number & Percent (\%) \\
\hline No Transformation & 20 & 64.50 \\
AML & 8 & 25.80 \\
ALL & 3 & 9.70 \\
\hline
\end{tabular}

performance score and lack of resources. Out of these 6 patients, none responded to induction chemo. Putting it all together, 9 out of 11 patients expired after transformation and 2 were lost to follow up.

\section{Discussion}

We conducted this study to identify the type of chromosomal aberrations apparent in our CML population right from the time of diagnosis and who happen to be in chronic phase, and response of such patients to the conventional TKIs. Dasatinib is not available in our country hence; Nilotinib was the second generation TKI that was offered as alternate drug in case of suboptimal response to Imatinib. The other aspect that we looked at was the risk of transformation to acute leukemias including both Acute Myeloid Leukemia and Acute Lymphoblastic Leukemia and their response to induction chemotherapy along with overall survival in such patients.

Chronic Myeloid Leukemia is a clonal myeloproliferative disorder with a characteristic reciprocal translocation $\mathrm{t}(9 ; 22)$ ( $\mathrm{q} 34 ; \mathrm{q} 11)$ resulting in Philadelphia chromosome [11] Additional chromosomal abnormalities are found in 10 to $20 \%$ of CML in chronic phase with incidence rising to as high as $80 \%$ in blastic phase [12]. Therefore, cytogenetic analysis plays a pivotal role not only in diagnosis and monitoring of response to therapy in CML but also as a prognostic indicator [13]. In our study it was depicted that $10.95 \%$ patients who were diagnosed with CML-CP had additional chromosomal abnormalities at the time of diagnosis. More common aberrations were Variant Philadelphia chromosome followed by trisomy 8 and Variant Philadelphia combined with other chromosomal aberrations. There has been a regional study on cytogenetic analysis in CML which showed secondary aberrations in $8.1 \%$ of cases, Variants of Philadelphia chromosome in $0.9 \%$ cases and $4.5 \%$ patients showed other chromosomal aberrations like +8 , del 20q and del 11q [13]. Another study done in Egypt showed that extra Philadelphia chromosome, trisomy
8 , isochrome $17 \mathrm{q}$ and trisomy 19 are most common additional chromosomal abnormalities [14].

Imatinib, a tyrosine kinase inhibitor targeting BCRABL fusion gene was introduced 10 years back and forms the main stay of treatment for CML with induction of complete cytogenetic response in more than $80 \%$ of newly diagnosed patients with CML-CP [5]. Despite great results in most of the cases, there have been suboptimal results in few [11]. CML with additional chromosomal aberrations are associated with poor response to TKIs and adverse survival [15-16]. Our study results also showed that while $51.6 \%$ patients achieved early complete hematological response within 3 months, more than half of the patients failed to achieve a complete molecular or cytogenetic response with tyrosine kinase inhibitor therapy.

Our study showed a risk of transformation to acute leukemia in $35.5 \%$ of patients. $27.3 \%$ of transformed patients had acute lymphoblastic leukemia whereas the rest of them were diagnosed to be having acute myeloid leukemia. Of the 11 transformed patients, only 6 were given induction chemotherapy and they failed to show any response. The remaining 5 patients were not considered fit for it mainly due to poor performance score.

In conclusion, the purpose of this project was to study the clinical presentation of patients with CML harboring additional chromosomal abnormalities at diagnosis, cytogenetic analysis, risk of blastic transformation and response to treatment in transformed patients. Despite an early hematological response, more than half of the patients failed to achieve complete molecular and cytogenetic response which clearly shows suboptimal response to the tyrosine kinase inhibitors in this particular set of CML patients. There was transformation to acute leukemia in $35.5 \%$ of patients. We were able to give induction chemotherapy to only 6 out of 11 patients and none of them responded to it. This study showed that presence of ACA at the time of diagnosis is a high-risk feature for patients with CML and confers poor prognosis when treated with conventional TKI. Further studies are required in our population regarding alternative therapy for such patient population.

\section{Acknowledgements}

I would like to thank my mentors Dr Neelam Siddiqui and Dr Abdul Hameed for their tremendous guidance in completion of this project. 


\section{Abbreviations}

CML, Chronic Myeloid Leukemia; CP, Chronic Phase; TKIs, Tyrosine Kinase Inhibitors; ACAs, Additional chromosomal abnormalities; AML, Acute Myeloid leukemia; ALL, Acute Lymphocytic Leukemia.

\section{Authors}

* The study was done at Shaukat Khanum Memorial Cancer Hospital and Research Centre, Lahore, Pakistan and all four authors were there at that time.

\section{References}

1. Perrotti D, Jamieson C, Goldman J, Skorski T. Chronic myeloid leukemia: mechanisms of blastic transformation. Journal of Clinical Investigation. 201007 01;120(7):22542264. https://doi.org/10.1172/jci41246

2. Crisan A, Coriu D, Arion C, Colita A, Jardan C. The impact of additional cytogenetic abnormalities at diagnosis and during therapy with tyrosine kinase inhibitors in Chronic Myeloid Leukaemia. J Med Life. 2015;8(4):502-8.

3. Kim TD, Turkmen S, Schwarz M, Koca G, Nogai H, Bommer C, Dorken B, Daniel P, le Coutre P. Impact of additional chromosomal aberrations and BCR-ABL kinase domain mutations on the response to nilotinib in Philadelphia chromosome-positive chronic myeloid leukemia. Haematologica. 2009 Dec 16;95(4):582-588. https://doi.org/10.3324/haematol.2009.014712

4. Mohamed A, Pemberton P, Zonder J, Schiffer C. The effect of imatinib mesylate on patients with Philadelphia chromosome-positive chronic myeloid leukemia with secondary chromosomal aberrations. Clin Cancer Res. 2003;9(4):1333-7.

5. Otero L O, de Azevedo AM, Dobbin J, Abdelhay E, Bouzas LF, et al. The impact of additional chromosomal abnormalities in response to Imatinib Mesylate therapy for Chronic Myeloid Leukemia Revista Brasileira de Cancerologia. 2007. 2007;53:405-10.

6. M. Greulich-Bode K, Heinze B. On the Power of Additional and Complex Chromosomal Aberrations in CML. Current Genomics. 201208 01;13(6):471-476. https://doi. org/10.2174/138920212802510466

7. Bennour A, Tabka I, Ben Youssef Y, Kmeira Z, Khelif A, Saad A, et al. A novel $\mathrm{t}(3 ; 12)(\mathrm{q} 21 ; \mathrm{p} 13)$ translocation in a patient with accelerated chronic myeloid leukemia after imatinib and nilotinib therapy. Cancer Biol Med. 2013;10(1):47-51.

8. Syed NN, Usman M, Adil S, Khurshid M. Additional chromosomal abnormalities in Philadelphia-positive chronic myeloid leukemia. Hematology/Oncology and Stem Cell Therapy. 2008 07;1(3):166-170. https://doi.org/10.1016/ s1658-3876(08)50025-2

9. Sgherza N, Abruzzese E, Perla G, Minervini MM, Chiello V, Sciannamè N, Cascavilla N. Onset of chronic myeloid leukemia with complex karyotype in a pregnant patient: case report and revision of literature. Therapeutics and Clinical Risk Management. 2017 06; Volume 13:751-755. https://doi. org/10.2147/tcrm.s137103

10. Verma D, Kantarjian H, Shan J, O'Brien S, Estrov Z, Garcia-Manero G, Koller C, Borthakur G, Cortes J. Survival outcomes for clonal evolution in chronic myeloid leukemia patients on second generation tyrosine kinase inhibitor therapy. Cancer. 2010;:NA-NA. https://doi.org/10.1002/ cncr. 25015

11. Luatti S, Castagnetti F, Marzocchi G, Baldazzi C, Gugliotta G, Iacobucci I, Specchia G, Zanatta L, Rege-Cambrin
G, Mancini M, Abruzzese E, Zaccaria A, Grimoldi MG, Gozzetti A, Ameli G, Capucci MA, Palka G, Bernasconi P, Palandri F, Pane F, Saglio G, Martinelli G, Rosti G, Baccarani M, Testoni N. Additional chromosomal abnormalities in Philadelphia-positive clone: adverse prognostic influence on frontline imatinib therapy: a GIMEMA Working Party on CML analysis. Blood. 201207 26;120(4):761-767. https:// doi.org/10.1182/blood-2011-10-384651

12. Aydin C, Cetin Z, Salim O, Yucel OK, Undar L, Berker Karauzum S. Previously Unreported Chromosomal Aberrations of $\mathrm{t}(3 ; 3)(\mathrm{q} 29 ; \mathrm{q} 23), \mathrm{t}(4 ; 11)(\mathrm{q} 21 ; \mathrm{q} 23)$, and $\mathrm{t}(11 ; 18)(\mathrm{q} 10 ; \mathrm{q} 10)$ in a Patient with Accelerated Phase $\mathrm{Ph}+$ CML. Case Reports in Genetics. 2014;2014:1-3. https://doi. org $/ 10.1155 / 2014 / 582016$

13. Chavan D, Ahmad F, Iyer P, Dalvi R, Kulkarni A, Mandava S, et al. Cytogenetic investigation in chronic myeloid leukemia: study from an Indian population. Asian Pac J Cancer Prev. 2006;7(3):423-6.

14. Eissa D, El Gendi H, Fouad D, Mohamed A, Mostafa N. Clinicopathologic features and prognostic impact of isochromosome $17 \mathrm{q}$ in chronic myeloid leukemia patients. The Egyptian Journal of Haematology. 2016;41(1):9. https:// doi.org/10.4103/1110-1067.178464

15. Hsiao H, Liu Y, Tsai H, Hsu J, Yang W, Chang C, Lin S, Xiao Huihua, Liu Yichang, Cai Huizhen, Xu Ruifeng, Yang Wenqi, Zhang Zhaosong, Lin Shengfeng. Additional chromosome abnormalities in chronic myeloid leukemia. The Kaohsiung Journal of Medical Sciences. 2011 02;27(2):49-54. https:// doi.org/10.1016/j.kjms.2010.09.001

16. Wang W, Cortes JE, Tang G, Khoury JD, Wang S, BuesoRamos CE, DiGiuseppe JA, Chen Z, Kantarjian HM, Medeiros LJ, Hu S. Risk stratification of chromosomal abnormalities in chronic myelogenous leukemia in the era of tyrosine kinase inhibitor therapy. Blood. 201606 02;127(22):2742-2750. https://doi.org/10.1182/ blood-2016-01-690230

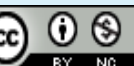

This work is licensed under a Creative Commons AttributionNon Commercial 4.0 International License. 